\title{
I federalisti italiani e francesi di fronte a Charles de Gaulle
}

Les fédéralistes italiens et français face à Charles de Gaulle

Italian and French Federalists facing Charles de Gaulle

\section{Raffaella Cinquanta}

\section{OpenEdition \\ Journals}

Edizione digitale

URL: http://journals.openedition.org/cei/2849

DOI: 10.4000/cei.2849

ISSN: 2260-779X

Editore

UGA Éditions/Université Grenoble Alpes

\section{Edizione cartacea}

Data di pubblicazione: 20 avril 2016

Paginazione: 61-75

ISBN: 978-2-84310-323-0

ISSN: $1770-9571$

Notizia bibliografica digitale

Raffaella Cinquanta, «l federalisti italiani e francesi di fronte a Charles de Gaulle», Cahiers d'études italiennes [Online], 22 | 2016, online dal 01 janvier 2017, consultato il 27 mars 2021. URL: http:// journals.openedition.org/cei/2849 ; DOI: https://doi.org/10.4000/cei.2849 


\title{
I FEDERALISTI ITALIANI E FRANCESI DI FRONTE A CHARLES DE GAULLE
}

\author{
Raffaella Cinquanta \\ Università degli Studi dell'Insubria
}

Dopo la bocciatura, nel 1954, del progetto di Comunità europea di difesa (Ced) e di quello, correlato, di Comunità politica europea (Cpe), per i federalisti europei militanti nei movimenti nati durante la Seconda guerra mondiale e raggruppati in una struttura di coordinamento, l'Unione europea dei federalisti (Uef) ${ }^{\text {, }}$, la Francia della Quarta Repubblica costituiva il principale ostacolo sulla via della creazione della Federazione europea ed era pertanto oggetto di particolare attenzione. Ciò non solo e non tanto perché era stata proprio l'Assemblea nazionale d'Oltralpe ad aver provocato la caduta della Ced, ma soprattutto perché la debolezza politica dei governi francesi e la pessima gestione delle situazioni di crisi - quali quelle in Indocina, in Algeria, e soprattutto di Suez - costituivano il più vistoso sintomo dell'inadeguatezza della formula dello Stato-nazione. A fortiori dopo la caduta della Repubblica nel maggio del 1958, quando - come sottolineava uno dei padri del federalismo, Altiero Spinelli — avendo scelto con la nomina di Charles de Gaulle alla presidenza del Consiglio di «gettarsi sul folle cammino della falsa grandezza nazionale [...] con la pretesa di occupare da sola il ruolo di fattore autonomo nell'equilibrio mondiale che solo l'Europa unita potrebbe occupare», la Francia sembrava rimettere in discussione il «valore delle istituzioni democratiche» ${ }^{2}$. Il problema del superamento della dimensione nazionale dello Stato si legava quindi ora

I. Per un inquadramento di questi temi si segnalano i lavori di U. Morelli, L'Unione europea dei federalisti e il Movimento federalista europeo sovranazionale, e di J.-P. Gouzy, I movimenti per l'unità europea in Francia, entrambi in S. Pistone (a cura di), I movimenti per l'unità europea 1954-1969, Pavia, Pime, 1996, pp. 55-69 e pp. $179-227$.

2. Archivio storico dell'Università di Pavia, Fondi aggregati (d'ora in poi ASUP/FA), Fondo Mfe (d'ora in poi MFE), 9/5, Lettera di A. Spinelli ai membri del Cc del Mfe, 20/09/1958. 
anche a quello della salvaguardia della democrazia, in Francia e, in subordine, in tutta Europa. I fatti, per coloro la cui memoria del conflitto mondiale era ancora fresca, non potevano non destare preoccupazione; rilevava eloquentemente Spinelli: «Si sta verificando, come nel passato (Mussolini, Hitler) la "corte all'uomo forte" "3. I federalisti si rendevano conto che fosse necessario, dopo la delusione del 1954, ripensare la propria politica e ideare le azioni necessarie a far fronte a tale situazione, nuova e inquietante, e sapevano di doverlo fare in modo unitario.

Ma ciò non era di facile attuazione; ci si propone pertanto in questa sede di analizzare, rispetto a tale questione, i rapporti molto difficoltosi tra la componente italiana e quella francese dell'Uef, al tempo le più consistenti. I due movimenti infatti, pur condividendo il giudizio negativo sul nazionalismo, presentavano differenti visioni politiche sia a livello teorico che pratico: se quella francese, 'proudhoniana', si batteva per il federalismo infra-nazionale, volto alla definizione di un nuovo ordine sociale ed economico oltre che politico - tale corrente era detta del 'federalismo integrale' e il suo principale esponente era Alexandre Marc ${ }^{4}$ - quella italiana, guidata da Altiero Spinelli e poi da Mario Albertini, si manteneva invece rigorosamente 'hamiltoniana', ovvero fautrice del federalismo sovranazionale ed istituzionale. Non mancavano divergenze anche a livello di azione: tendenzialmente moderato e, in alcune sue componenti filogovernativo, il Movimento francese ${ }^{5}$, più radicale, antigovernativo, intenzionato a fare dei federalisti una forza politica completamente autonoma, quello italiano. Le difficoltà erano esplose proprio quando i federalisti italiani erano stati costretti, nel 1956-1957, a dar vita all'iniziativa per la convocazione di un'Assemblea costituente europea, il Congresso del popolo europeo (Cpe), al di fuori dell'Uef, provocando un contrasto tale al suo interno da indurre alla scissione alcune sue componenti, che poi formarono un'organizzazione alternativa, l'Action européenne fédéraliste (Aef) ${ }^{6}$. Tuttavia,

3. ASUP/FA, MFE, 9/4, Verbale del Cc Mfe, I4-15/06/1958.

4. Cfr. A. Marc, Du communalisme au fédéralisme intégral, Paris, La Fédération, s. d. [1948]; Id., La Révolution fédéraliste, Paris, Presse d'Europe, 1969; Id., Europa e federalismo globale, Firenze, Il ventilabro, 1996.

5. Una concausa di tale moderatismo è la coeva presenza in Francia di molte organizzazioni europeiste dal forte peso politico, tra le quali, oltre al Comitato d'azione per gli Stati Uniti d'Europa di Jean Monnet, vanno citate quelle raggruppate nell'Organizzazione francese del Movimento europeo (Me), come La Fédération di André Voisin — sostanzialmente di destra — e il Movimento socialista per gli Stati Uniti d'Europa dell'exministro Gérard Jaquet.

6. L'idea del Cpe fu concepita nel 1955 (cfr. A. Spinelli, Diario europeo, a cura di E. Paolini, vol. I (1948I969), Bologna, Il Mulino, I989, p. 240), approvata dal Cc del Mfe italiano nel 1956 («Europa Federata», d'ora in poi $\mathrm{EF}$, vol. IX, $\mathrm{n}^{\circ}{ }^{\mathrm{I} 2}$, I956, p. 4) e fu la causa del contrasto con le componenti tedesca e olandese dell'Uef, che se ne distaccarono e con La Fédération diedero vita all'Aef. Cfr. anche G. Héraud, Che cos’è il CPE, «Popolo Europeo» (d'ora in poi PE), vol. IV, nº 8, I96I, p. 3. 
al di là delle differenze dottrinali, il problema di base dell'Uef stava in realtà nella sua struttura, non centralizzata e di mero coordinamento tra movimenti nazionali pienamente indipendenti a livello politico: se ciò rendeva difficile trovare linee di azione comune nella norma, condannando il Movimento, nel suo complesso, a una semicronica debolezza politica, diventava del tutto paralizzante in momenti di crisi. Ciò si verificò puntualmente proprio a seguito della comparsa sulla scena politica di Charles de Gaulle. Come aveva percepito Spinelli, «la tentazione poteva essere forte di restare intimoriti dal confronto tra la nostra debolezza e il peso della politica francese» ${ }^{7}$, e infatti, al di là delle iniziative dei singoli movimenti nazionali, l'Uef nel suo complesso si ritrovò, per indecisione, dispersione di forze, e soprattutto per mancanza di una strategia politica elaborata direttamente a livello sovrannazionale, ad arrancare inseguendo gli eventi, piuttosto che a indirizzarli, come invece era avvenuto negli anni della Ced.

Nell'analisi di questa intricata situazione è parso affiorare un rapporto di derivazione piuttosto diretto tra l'evoluzione politico-organizzativa che l'Uef sperimentò, proprio nel tentativo di porre rimedio a tali mancanze, e i cambiamenti di rotta della politica gollista; parrebbe quindi ragionevole affermare che quest' ultima, per le caratteristiche che si vedranno, 'obbligò' il Movimento a rientrare nell'agone politico e a rinnovarsi completamente. Rispetto a questo rapporto di derivazione, sono state riscontrate due fasi. Nella prima, che va dal 1957 al 1962, gli eventi politici francesi contribuirono a mantenere unito il Movimento - che, forse, sotto la pressione del Mfe-Italia, si sarebbe altrimenti disgregato — inducendolo a ristrutturarsi in organismo centralizzato a livello sovrannazionale e, di conseguenza, obbligandolo anche ad abbozzare una linea di compromesso politico tra le correnti. Compromesso che però non trovò un corrispondente a livello di azione; e le ragioni di ciò sono in gran parte attribuibili proprio alla politica gollista, sotto due specifici aspetti. Da una parte, essa sembrava esercitare, nei suoi primi anni, un'indubbia 'seduzione' sull'intimorito cittadino francese, seppur federalista. Spinelli ne coglieva il punto fondamentale, quando sottolineava che, se di fronte a de Gaulle non scattava l'istinto democratico, bensì quello del cittadino bisognoso di quiete e ordine, il Generale poteva facilmente apparire come colui che non solo proteggeva la Francia dalle dittature fascista e comunista, ma lo faceva, nel contempo, attraverso una riproposizione dell'idea europea ${ }^{8}$. Ma vi era

7. A. Spinelli, Diario europeo, cit., p. 358.

8. Ibid. 
anche un secondo, più sottile, aspetto, che è forse l'elemento chiave per comprendere le ragioni per le quali la figura di de Gaulle riuscì a confondere, inaspettatamente, anche i federalisti che non lo sostenevano. Come infatti notava Mario Albertini nel $196 \mathrm{I}^{9}$, de Gaulle aveva il merito di aver compreso quel che non volevano comprendere i fautori dell'Europa comunitaria, ovvero che l'unità dell'Europa era un problema di natura eminentemente politica. Erano politiche infatti le argomentazioni addotte in difesa dell'autonomia dell'Europa rispetto agli USA — simboleggiate dal veto all'entrata della Gran Bretagna del I4 gennaio I963 - del suo ruolo di potenza economica e di punto di riferimento alternativo ai due blocchi, così come avevano finalità politiche, seppur di natura confederale, i Piani Fouchet. Coloro che intendevano svolgere opposizione al suo progetto, politico, di Europa confederale erano quindi anch'essi obbligati a farlo, e concretamente, sul piano politico; dichiarazioni d'intenti e professioni dottrinali non erano più sufficienti, bisognava agire; diversamente, si era condannati all'impotenza. E impotente era infatti l'Uef, frenata dal Movimento francese, a sua volta bloccato tra l'ostruzionismo dei suoi membri gollisti e il disorientamento o la cautela degli altri. Da tutto ciò l'organizzazione fu però strappata a forza nella seconda fase, compresa tra la seconda metà del 1962 e il 1969, quando fu spinta a «uscire dai suoi soliloqui per incontrarsi e scontrarsi con i gruppi di potere e di interesse proprio sul terreno dell'Europa ${ }^{\text {ㅇ․ }}$. Il cambio di rotta della politica europea gollista, che da propositiva divenne completamente ostruzionistica, creò infatti una situazione emergenziale tale far approdare definitivamente il Movimento francese su posizioni di aperta opposizione e, in un secondo momento, da far convergere tutte le forze federaliste ed europeiste in funzione antigollista. La minaccia alle istituzioni europee, peraltro, avveniva proprio nel momento in cui il mercato comune registrava innegabili successi; tale convergenza fu attuata quindi sulla base di una piattaforma politica che consentiva di integrare diversi approcci, anche non rigorosamente federalisti, grazie a due dispositivi pratici di immediata fruizione - il Fronte democratico e l'elezione diretta del Parlamento europeo - i quali, se da una parte utilizzavano forze e mezzi disponibili nell'hic et nunc, dall'altra spianavano la strada ad obiettivi più ambiziosi per il futuro. E sarà questa piattaforma — alla quale Albertini affiancherà più avanti la teoria

9. M. Albertini, Quattro banalità e una conclusione sul vertice europeo, «Il Federalista» (d'ora in poi IF), vol. III, $\mathrm{n}^{\circ}$ 2, I96I, pp. $63 \mathrm{sgg}$.

Io. Historical Archives of the European Union (d'ora in poi HAEU), AS/ooso, Lettera di O. Giarini ad A. Spinelli, 23/09/1962. 
politologica del 'gradualismo' II — che consentirà nel decennio successivo ai federalisti di riacquistare un forte peso politico e un'influenza consistente sugli eventi, tradottesi nelle lotte per l'elezione diretta del Pe e per lo Sme.

\section{Riforma istituzionale e compromesso politico (I957-1962)}

Nella prima fase, a fronte della paralisi del Movimento francese, il ruolo propulsivo fu svolto da quello italiano, che aveva definito suo obiettivo primario post 1954 lo svolgimento di una strenua opposizione, in particolare al nuovo regime francese ${ }^{\mathrm{I} 2}$, nella speranza di poterne sfruttare le contraddizioni in prospettiva federale. Spinelli dichiarava infatti in un articolo del giugno 1958 , Il nostro fronte ${ }^{13}$, che la «missione storica» del popolo francese era di battersi affinché la rinascita della democrazia francese fosse «l'atto di nascita della democrazia europea», assegnando a ciò una tale importanza da sostenere che l'avvenire della sua azione ormai ne dipendeva completamente ${ }^{\mathrm{I}}$. Come strumento Spinelli puntava sul Cpe, che avrebbe dovuto creare un suo fronte francese per la lotta sia contro de Gaulle che contro la restaurazione democratica su base nazionale. Già nel maggio del 1958 quindi il Congresso votava una risoluzione di dura condanna del nazionalismo francese, mentre nel luglio successivo prendeva una chiara posizione sulla nuova costituzione seguita in settembre da un appello ai francesi affinché votassero no al referendum ${ }^{15}$.

Spinelli forzò molto la mano in sede Uef affinché si allineasse con il Cpe. Dalla sua parte si schierarono - con differenze di grado e momenti di ripensamento ma pur sempre nella convinzione che anche il federalismo integrale necessitasse di uno slancio di tipo popolare, attivabile grazie alla rivendicazione costituente - Michel Mouskheli, che diventerà presidente del Cpe, e Alexandre Marc, che, allora presidente del Bureau exécutif $(\mathrm{Be})$ dell'Uef, entrò anche nel Comitato permanente del Cpe. Il principale oppositore era invece Henri Frenay, leader del Mfe francese, che divenne apertamente filo-gollista e che era seguito, spesso anche al

II. Cfr. i testi di M. Albertini, Elezione europea, governo europeo, stato europeo (IF, vol. XVIII, n ${ }^{4}$, I976, p. 200 sgg.) e Rapporto di Mario Albertini al Comitato federale dell'Uef del I8-19/02/1978 (IF, vol. XX, nº I, I978, p. I sgg.).

I2. Lettera di A. Spinelli ai membri del Cc del Mfe, 20/09/1958, cit.

I3. PE, vol. I, no 6, I958, p. I.

I4. A. Spinelli, Diario europeo, cit., p. 348.

I5. Vedi, rispettivamente, PE, vol. I, I958, $\mathrm{n}^{\circ}$ 5, p. I; $\mathrm{n}^{\circ} 7$, p. I e $\mathrm{n}^{\circ} 8$, p. I. 
solo fine di evitare contrasti al suo interno, dalla maggior parte del Mfe d'Oltralpe, anche da chi, come Jean-Pierre Gouzy e André Delmas, erano orientati favorevolmente alle tesi italiane ${ }^{16}$. L'opposizione a Spinelli si manifestava, in sede Uef, nella riluttanza dei membri francesi a prendere posizioni chiare, e nell'assunzione di atteggiamenti dottrinali, mascherati dal pervicace attaccamento all'obiettivo del federalismo integrale; tuttavia, per evitare l'isolamento, premeva loro anche evitare che il Movimento si disgregasse, ed è per questo che cercarono sempre di scendere a compromessi con la componente italiana. Compromessi del resto sempre più al ribasso e ottenuti al prezzo dell'inerzia dell'organizzazione. Prova ne sia il fatto che, pur adottando nel 1957 la parola d'ordine della Costituente e pur esprimendo qualche apertura verso il $\mathrm{Cpe}^{17}$, nel $1958 \mathrm{fu}$ ribadito il rifiuto di farne propria l'azione ${ }^{18}$, con ciò peggiorando di fatto la situazione di entrambe le organizzazioni poiché, mantenendone scoordinati organi e funzioni, se ne sdoppiavano le azioni e indeboliva l'efficacia. Di tutto ciò prende impietosamente atto Spinelli quando, in occasione di una Conferenza Uef organizzata nel luglio del 1958 per esaminare la crisi francese ${ }^{19}$, commentava: «Il federalismo francese [...] per non aver mai voluto scegliere in modo definitivo tra il nazionalismo [...] e l'Europa [...] è ormai agonizzante, e con esso l'Uef ${ }^{20}$.

Fu del resto proprio la crisi francese, sempre più allarmante, a dotare l'ipotesi di un rinnovamento dell'Uef, quantomeno a livello procedurale e strutturale, del carattere dell'urgenza. Alcuni dirigenti, tra cui il belga Raymond Rifflet - moderato e mediatore tra italiani e francesi - e Luciano Bolis - trait d'union tra Uef, Mfe-Italia e Cpe - rilevavano l'opportunità che il Movimento esprimesse le sue linee politiche chiaramente, senza "vaines précautions diplomatiques»" ${ }^{21}$, e procedesse a «una modificazione dei $[\ldots]$ metodi di lotta ${ }^{22}$. Ciò divenne ineludibile proprio nel periodo a cavallo del referendum sulla Quinta Repubblica, quando, nonostante i federalisti italiani esprimessero la propria solidarietà fattiva a coloro che in Francia intendessero battersi contro de Gaulle, sperando così

I6. Il contrasto tra Spinelli e Frenay risaliva al 1955 ed esplose in occasione della scissione del 1956 (cfr. A. Spinelli, Diario europeo, cit., pp. 236-239, p. 290 e pp. 349-352).

17. ASUP/FA, Mfe, C/7/12, Compte rendu du BE UEF (02-03/11/1957) e Compte rendu du CC UEF (I4I5/I2/I957).

18. ASUP/FA, Mfe, C/7/I2, Compte rendu du BE UEF (oI-02/02/1958).

19. Vedi EF, vol. XI, no $8-9$, I958, p. 2.

20. A. Spinelli, Diario europeo, cit. p. 357.

2I. HAEU/RR/75, Lettera di R. Rifflet alla Commissione nazionale francese del Mfe, 29/II/I959.

22. ASUP/FA, MFE, C/6/4, Lettera di L. Bolis a G. Usellini, 3I/05/1958. 
di dar loro coraggio ${ }^{23}$, l'Uef aveva mostrato di «non avere né sufficiente volontà politica europea né la capacità di assumere responsabilità politiche importanti ${ }^{24}$, votando risoluzioni che non prendevano una posizione decisa sul regime gollista e non rilevavano né l'urgenza né - cosa ancora peggiore - la necessità di un intervento, limitandosi a raccomandare una generica vigilanza ${ }^{25}$. Il fatto poi che il Mfe francese avesse lasciato i suoi membri liberi di votare secondo coscienza al referendum, costituiva una riprova del fatto che l'Uef era politicamente pietrificata. Il Mfe italiano tentò di porre rimedio a tale situazione sia convocando la seconda sessione del Cpe proprio in Francia, a Lione - una delle poche roccaforti francesi del federalismo radicale e in occasione della quale fu approvato un progetto di Trattato per la convocazione della Costituente ${ }^{26}$ — che rivolgendo, negli stessi giorni del referendum, «un estremo appello» al Comitato centrale $(\mathrm{Cc})$ dell'Uef affinché si trasformasse in movimento unitario e sostenesse il $\mathrm{Cpe}^{27}$ — obiettivi, secondo Spinelli, rigorosamente consequenziali per "affrontare il prossimo periodo difficilissimo» ${ }^{28}-$ arrivando a minacciare anche la scissione. I francesi, pur cercando di mediare, erano riluttanti, forse anche nel timore che un organismo centralizzato avrebbe implicato una subordinazione al Mfe italiano, ma l'elezione di de Gaulle alla presidenza della Repubblica fece mutare loro opinione: Spinelli testimoniava infatti che la forte presa di posizione del Mfe-Italia, in unione però al «rafforzarsi del regime di de Gaulle, nei cui confronti sono cadute molte speranze di federalisti francesi», aveva «spinto a un rinnovamento» ${ }^{29}$. Nel gennaio del 1959 l'Uef nominava quindi una Commissione per esaminare il problema della sua conversione in organismo unitario ${ }^{30}$; ciò avvenne al Congresso straordinario di Parigi del 27-29 giugno successivo, quando l'Uef si trasformò in Mfe-sovrannazionale, organizzazione organica a gestione centralizzata, mentre i movimenti nazionali diventavano semplici strutture amministrative, le Commissioni nazionali ${ }^{31}$. Frenay e i gollisti decisero di farsi da parte e ciò ebbe come immediato effetto una collaborazione più stretta con il Cpe: onde evitare la dispersione di forze e

23. EF, vol. XI, no 7 , I958, p. I.

24. Lettera di Spinelli ai membri del CC del Mfe, 20/09/1958, cit.

25. Vedi ad esempio ASUP/FA, MFE, C/7/12, Les États-Unis d'Europe : un objectif pour la nouvelle constitution française. Résolution du CC UEF, 23/06/1958.

26. PE, vol. II, n ${ }^{\circ}$ I2, I959, p. I.

27. Resoconto del CC Mfe, 27-28/09/1958, EF, vol. XI, nº II, I958, p. I.

28. ASUP/FA, MFE, C/6/4, Lettera di A. Spinelli a G. Usellini, I9/o9/1958.

29. ASUP-FA, MFE, 9/6, Verbale del CC Mfe, II-I2/O4/I959.

30. La documentazione relativa ai lavori della Commissione è in ASUP/FA, Mfe, C/6/4 .

3I. Cfr. G. Héraud, Che cos’è il Mfe, PE, vol. IV, n 6-7, I96I, p. 3. 
iniziative si decise infatti di invitare i rispettivi dirigenti ad assistere alle relative riunioni ${ }^{32}$, mentre il Comitato permanente del Cpe cooptava Delmas, nuovo Segretario generale del Mfe, Rifflet e Gouzy ${ }^{33}$. Quest'ultimo poi, nuovo Segretario della Commissione nazionale francese, in una lettera ai lettori del foglio del Cpe, "Popolo europeo», sottolineava significativamente come i federalisti dell'Uef appartenessero a una tendenza "sorella» che, pur essendo rimasta per lungo tempo nell'aspettativa giudicando troppo «ambiziosa» l'azione del Cpe, avrebbe potuto essere finalmente convinta a superare le ultime esitazioni ${ }^{34}$.

Ma aver riformato la propria struttura e aver allontanato i gollisti non significava ancora aver rinnovato la propria politica. Fu così che, ai due successivi Congressi (Strasburgo, 29-3I gennaio I960, e Lione, 9-II febbraio 1962) ${ }^{35}$ fu faticosamente tratteggiata una linea basata sulla strategia costituente che, prevedendo sia la redazione di una Carta politica come documento programmatico di base per la definizione delle azioni, cara al federalismo francese, che la fusione tra Mfe e Cpe, accontentava entrambe le fazioni ${ }^{36}$. Le quali furono peraltro concordi sull'adozione di un nuovo orientamento, meno intransigente, proposto da Spinelli ${ }^{37}$ : la ricerca di un'alleanza 'critica', in quanto forza europea autonoma, con i gruppi di opposizione democratica, opposizione «ancora informe e assai mal rappresentata", da conquistare alla causa europea proprio a partire dalla Francia ${ }^{38}$. Inoltre, com'era prevedibile, maggior unità politica comportò maggior audacia politica; furono quindi votate due chiare risoluzioni: a Strasburgo sull'Algeria e a Lione contro de Gaulle ${ }^{39}$. Quest' ultima, in particolare, elaborata dopo un faticoso compromesso sulla base di un documento 'canovaccio' presentato da Rifflet, assegnava al regime francese la precisa responsabilità del degenerare della situazione ${ }^{40}$ e fu seguita da un comunicato del Be nel quale si dichiarava come idea d'Europa gollista e Federazione

32. ASUP/FA, MFE, C/7/12, Compte rendu du CC MFE, 26-27/09/1959.

33. PE, vol. II, $\mathrm{n}^{\circ} 23$, I959, p. 3.

34. PE, vol. III, $\mathrm{n}^{\circ} \mathrm{I}$, I960, p. 3 .

35. Sui Congressi vedi PE, vol. III, $\mathrm{n}^{\circ}$ 2, 1960 e vol. V, $\mathrm{n}^{\circ} 2,1962$.

36. A fine I960 le prime elezioni dei delegati al Cpe in Francia si svolsero con successo a Lione e ad Annecy (PE, vol. III, nº II, I960, p. I).

37. Per questo cambio di rotta si scontrò con Albertini, dando il via alle difficoltà che sempre caratterizzeranno i loro rapporti politici (Cfr. A. Spinelli, Diario europeo, cit., p. 420).

38. A. Spinelli, A Lione: il MFE e il CPE ad un bivio, PE, vol. V, $\mathrm{n}^{\circ} \mathrm{I}, \mathrm{I} 962, \mathrm{p} .8$.

39. Quanto all'Algeria, si tenga presente che non più tardi di un anno prima il BE non era riuscito a votare che una timida risoluzione, ove peraltro si sosteneva che il problema algerino fosse di competenza giuridica esclusivamente francese (Compte rendu du BE UEF, OI-02/02/1958, cit.).

40. Cfr. PE, vol. V, nº 2, 1962, p. 7. 
europea fossero due concezioni inconciliabili ${ }^{41}$. Da ultimo, va ricordata la decisione, presa dal Mfe nel I96I, di svincolarsi dal Movimento europeo $(\mathrm{Me})$, organizzazione genericamente europeista e filo-governativa, decisione che rientra nello sforzo di acquisizione di una maggior individualità e indipendenza politica ${ }^{42}$.

Tuttavia, al di là delle decisioni congressuali, nella pratica rimanevano difficoltà di non poco conto: «la sopravvivenza dell'organizzazione è affidata all'iniziativa slegata di pochi gruppi». Si registrava poi «un'estrema dispersione di atteggiamenti e di iniziative» ${ }^{43}$, ai cui estremi si ponevano ora, da un lato, l'opposizione intransigente organizzata nella corrente di Autonomia federalista — guidata da Mario Albertini, erede del radicalismo spinelliano ma con questi in contrasto e che, dopo vari conflitti, condurrà il Movimento negli anni Settanta ${ }^{44}$ - e dall'altro, la componente filo-comunitaria, guidata dal francese Germain Desbœuf, favorevole a un'azione di propaganda su governi e politici in collaborazione con il Me. Era quindi necessario andare oltre i compromessi e costruire un'effettiva unità, d'intenti e d'azione, senza la quale non era possibile effettuare il salto di qualità decisivo: l'elaborazione di politiche che non fossero il risultato del semplice affiancamento di tradizioni politiche nazionali ma di una vera e propria azione sovrannazionale concertata.

\section{Unità e azione (1962-1969)}

Inizia così la seconda fase dello sviluppo del Movimento, durante la quale le iniziative e il cambiamento radicale della politica europea di de Gaulle dopo la bocciatura dei Piani Fouchet - la crisi della sedia vuota, il compromesso di Lussemburgo, la politica in sede Nato, la force de frappe, il secondo, del tutto pregiudiziale, veto all'entrata della Gran Bretagna nella Cee - provocarono un tale stato di allerta nel milieu federalista ed europeista da spingere tutte le sue componenti verso la ricomposizione delle divergenze e l'aggregazione operativa.

4I. HAEU, RR/49, Communiqué de presse, s. d. [maggio 1962]. Da qui in avanti gli articoli a firma di federalisti francesi sui fogli del Movimento sono più scopertamente antigollisti.

42. PE, vol. IV, $\mathrm{n}^{\circ}$ 6-7, I96I, p. 3. È indicativo al riguardo che mentre il Mfe italiano aveva ritirato la sua delegazione già dal 1956 (EF, vol. IX, n²0, 1956, p. 4), l'Uef aveva deciso di non seguirlo finché non si fosse dotata di una politica efficace e condivisa da tutte le sue componenti (ASUP/FA, MFE, C/7/12, Compte rendu du BE UEF, 20-21/07/1957).

43. Resoconto del CC Mfe, 23-24/o6/1962, PE, vol. V, no 6-7, 1962, p. 7.

44. Sulla posizione di Autonomia federalista vedi M. Albertini, Le IXe Congrès du MFE. Introduction, IF, vol. IV, $\mathrm{n}^{\circ}$ I, I962, pp. 28 sgg. 
In prima battuta è tuttavia opportuno ricordare che tale sviluppo fu in un certo senso facilitato anche dal disimpegno di Spinelli dalle cariche più importanti del Movimento. Il suo fu un allontanamento graduale, iniziato proprio nel 1962 - a cavallo dei Piani Fouchet e dopo la presa d'atto che il Cpe, nonostante l'avvallo del Mfe-sovrannazionale, non stava ottenendo i risultati auspicati ${ }^{45}$ - sulla base di due importanti considerazioni, stimolate proprio dalla figura di de Gaulle. La prima era una costatazione: la politica europea del Generale aveva reso necessaria l'assunzione di posizioni chiare non tanto sulla necessità o meno dell'integrazione - cosa che de Gaulle stesso condivideva - bensì su quale Europa s'intendeva costruire: un'Europa autoritaria o un'Europa democratica ${ }^{46}$. L'Europa comunitaria infatti, proprio perché priva di individualità politica e non sostenuta da un progetto alternativo a quello gollista, poteva essere facilmente strumentalizzata al fine di assommare i vecchi nazionalismi in un nuovo nazionalismo europeo, che avesse come fine la politica della pura potenza e l'autoritarismo ${ }^{47}$. L'europeismo' gollista - e questa è la seconda, importante, considerazione - rischiava quindi di creare un'identificazione politica tra opposizione al suo regime e opposizione all'unità europea ${ }^{48}$. Tutto ciò richiedeva di agire con urgenza sulle forze che aspiravano a un rinnovamento; per Spinelli, il Mfe non era più la sede adatta, perché le linee direttive faticosamente definite non riuscivano a tradursi in azioni efficaci ${ }^{49}$ e scelse quindi una via alternativa, che lo condurrà alla collaborazione con il ministro degli Esteri italiano Pietro Nenni, in seguito alla Commissione europea, e infine al Pe. In sede Mfe il suo disimpegno consentì alla linea moderata francese di prevalere e all'organizzazione di assestarsi.

Ciò premesso, è possibile suddividere ulteriormente il periodo in tre parti, distintamente scandite dalle iniziative golliste. La prima si snoda tra la seconda metà del 1962 e il 1965 e vede la definitiva presa di coscienza, da parte del Mfe francese, dell'antieuropeismo della politica europea del Generale. A fungere da spartiacque in questo senso fu la sua conferenza stampa del I5 maggio I962, nella quale attaccava le tesi in favore della sovrannazionalità e criticava la struttura dell'integrazione atlantica. A tal riguardo non necessitano commenti le parole di Gouzy:

45. Cfr. A. Spinelli, Diario europeo, cit., p. 422.

46. Cfr. A. Spinelli, Europa fra imperialismo e democrazia, PE, vol. VI, nº 3, I963, p. 2.

47. ASUP/FA, Fondo Luciano Bolis (d'ora in poi LB), 57, Dichiarazione politica del CIDE, s. d. [1964].

48. Cfr. A. Spinelli, Una politica europea per le forze democratiche, PE, vol. VI, $n^{\circ} 5$ bis, I963, pp. 6-8.

49. Cfr. ASUP/FA, LB, 57, Lettera di A. Spinelli a L. Bolis, 02/06/1964. 
Il I5 maggio $1962[\ldots]$ il monarca che regge i destini della V Repubblica ha superato se stesso. [...] Tutti coloro che dal 1958 amoreggiano con il suo regime nella speranza di assistere a una evoluzione [...] hanno dovuto abbandonare questa illusione. [...] De Gaulle, sbarazzatosi dei fastidi più gravi prodotti dalla guerra in Algeria, [...], sta per mettere tutto se stesso al servizio del suo sostanziale nazionalismo ${ }^{50}$,

la cui più vistosa espressione sarà il Trattato dell'Eliseo del 22 gennaio 1963. Il Movimento francese usciva quindi dall'incanto e il Mfe poteva finalmente agire. La differenza rispetto alla cautela degli anni precedenti è immediatamente riscontrabile: i suoi organi decisero di pronunciarsi per il 'no' al referendum del 28 ottobre sull'elezione diretta del presidente della Repubblica — pur non schierandosi ancora apertamente con nessuna formazione politica ufficiale ${ }^{s \mathrm{I}}$ - e a tal proposito Gouzy si stupiva che «nessuno dei francesi presenti abbia questa volta messo in discussione l'opportunità di una tale decisione» ${ }^{52}$. Il fatto che un terzo dell'elettorato si astenesse dal votare, poi, fu interpretato come una prima avvisaglia della sfiducia dei francesi nel regime ma anche nella politica in generale, e ciò sembrava aprire nuovi spazi di intervento. Si profilava la possibilità di collaborazioni più vaste e proprio in tale contesto fece la sua comparsa l'idea tattica dell'elezione diretta del Pe quale mezzo «per una soluzione durevole alla crisi che oggi corrode le democrazie nazionali» ${ }^{53}$ : sulla sua base diventava infatti possibile riprendere i contatti sia con le altre componenti del federalismo europeo ${ }^{54}$ che con i partiti all'opposizione ${ }^{55}$ al fine di "costituire un'avanguardia in tutti i raggruppamenti politici», e invitare nel contempo tutte le organizzazioni animate da spirito europeo ad «associarsi a questo sforzo» ${ }^{56}$. Ripresero altresì le relazioni con il Me, che a sua volta iniziava a manifestare dissenso ${ }^{57}$; tutto ciò indusse la componente filocomunitaria del Mfe ad avvallare definitivamente la linea politica adottata.

Restavano tuttavia ancora da definire dei veri e propri dispositivi di azione pratica che permettessero al Movimento di passare da un ruolo reattivo agli eventi a uno propulsivo e attivo, di «catalizzatore delle energie europee sparse» ${ }^{58}$. Essi furono alfine individuati, sulla base del programma

50. J.-P. Gouzy, La crisi francese e il 'Volapuk' integrato, PE, vol. V, n 5, 1962, p. 3.

5I. PE, vol. V, $\mathrm{n}^{\circ}$ II, I962, p. 7.

52. J.-P. Gouzy, Il No coerente dei federalisti, PE, vol. V, $\mathrm{n}^{\circ}$ II, I962, p. 3.

53. Cfr. PE, vol. VI, n ${ }^{\circ}$ bis, I963, p. 9.

54. Cfr. ASUP/FA, LB, 57, Lettera di O. Giarini a L. Bolis, 24/05/1963.

55. Vedi HAEU, RR/5I, Lettera di J.-P. Gouzy a E. Hirsch, O. Giarini e R. Rifflet, I8/03/1963.

56. PE, vol. VI, $\mathrm{n}^{\circ} 7-8,1963$, p. 7. Dal 1964 in avanti si registrano infatti negli archivi materiali prodotti dagli altri movimenti e dalla Cee diffusi dal Mfe alle sezioni nazionali.

57. Cfr. PE, vol. V, n ${ }^{\circ}$ II, I962, p. 7.

58. Cfr. J.-P. Gouzy, Incertezze comunitarie e prospettive federaliste, PE, vol. VII, nº 5, I964, p. I. 
politico definito a Lione, al Congresso di Montreux del IO-I2 aprile $1964^{59}$. $\mathrm{Fu}$, in primo luogo, approvata la Carta federalista, un documento strutturato che inquadrava l'azione per la Federazione europea in un discorso più ampio di rinnovamento della società sulla linea del federalismo integrale, sulla base della quale si riuscì a unire le correnti del federalismo francese extra-Mfe, quali La Fédération di André Voisin e la Gauche européenne di André Philip. La Carta, che poneva quale obiettivo strategico l'Assemblea costituente, contemplava anche il sostegno alle iniziative della Commissione contro i tentativi «d'emprise» degli Stati nazionali e inglobava un appello a tutte le forze progressiste affinché si battessero per la Federazione ${ }^{60}$. Il secondo dispositivo, infatti, prevedeva la creazione di un Fronte federalista e democratico europeo, una forza di opposizione popolare ai nazionalismi in funzione antigollista ${ }^{61}$. Tale iniziativa, che riprendeva quanto già proposto da Spinelli e che quindi ne permise la partecipazione attiva, fu simboleggiata a Montreux dall'elezione alla presidenza del Cc del francese Étienne Hirsch, ex presidente dell'Euratom ed amico personale di Monnet — e, come dichiarava Spinelli stesso, «il personaggio chiave dell'operazione» ${ }^{62}-\mathrm{e}$ dalla risoluzione sulle elezioni presidenziali in Francia, nella quale si garantiva appoggio ai candidati a favore dell'Europa federata.

Nei mesi successivi, sulla base dell'idea del Fronte e a seguito della costituzione di appositi Comitati in Aquitania, a Lione e Parigi ${ }^{63}$, il Movimento seguì la Convenzione repubblicana di Parigi del 6-7 giugno 1964, che vide riuniti per la prima volta in forma ufficiale i rappresentanti dei Club - i circoli culturali determinati a dare un nuovo impulso alla sinistra democratica francese sotto la leadership di Mitterrand - e che produsse una dichiarazione conclusiva nella quale si rilevava come lotta per la difesa della democrazia ed Europa federale andassero di pari passo ${ }^{64}$. Ebbe anche inizio una proficua collaborazione con il Consiglio dei Comuni d'Europa (Cce) - in particolare con Umberto Serafini, fondatore della sua sezione italiana - che si concretizzò nella grande manifestazione federalista che furono i VII Stati generali di Roma del I5-I8 ottobre 1964 ${ }^{65}$. Rispetto all'idea del Fronte, tuttavia, Autonomia federalista si dichiarava critica, e,

59. Sul Congresso vedi PE, vol. VII, $\mathrm{n}^{\circ}$ 4, 1964.

6o. La Carta è in ASUP/FA, LB, 57.

6r. HAEU/AS/oi67, Lettera di A. Spinelli a E. Hirsch, Io/II/I964.

62. HAEU/AS/or67, Lettera di A. Spinelli a O. Giarini, o7/I2/1964.

63. "Giornale del Censimento" (d'ora in poi GC), vol. I, n ${ }^{\circ} 5$, 1965 , p. 2.

64. La Francia antigollista verso l'Europa, PE, vol. VII, n ${ }^{\circ}$ 6, I964, p. 2.

65. Cfr. PE, vol. VII, ${ }^{\circ}$ IO-II, I964, pp. 4-5. 
preferendo rimanere nel solco dell'iniziativa popolare costituente, optò per l'adozione dell'iniziativa del Censimento volontario del popolo europeo in sostituzione del $\mathrm{Cpe}^{66}$. Fronte e Censimento furono inizialmente svolti separatamente perché espressione della maggioranza e della minoranza del Movimento ${ }^{67}$, per poi essere pienamente coordinate nel giugno $1965^{68}$.

In tale occasione Mario Albertini pose infatti fine ai contrasti interni sciogliendo la corrente di Autonomia ed entrando nel Be; tra 1965 e 1967 - seconda sottosezione del periodo di riferimento - il Movimento ritrovò quindi una salda unità al suo interno, e conseguentemente acquisì più forza anche nei confronti dei movimenti esterni. Ma se si considera che il 1965 è anche l'anno in cui Albertini mutò opinione su de Gaulle, è forse possibile ipotizzare una connessione tra le due svolte, connessione direttamente riconducibile, nuovamente, alle azioni di quest'ultimo, in primis allo strappo alla Cee del 30 giugno 1965 e alla conferenza stampa del 9 settembre 1965 , nella quale si scagliò contro i Trattati comunitari ${ }^{69}$. Diversamente da quanto espresso nel 196I, gli albertiniani vedevano ora nel Generale niente più che una forza di ostruzione - «il suo ruolo storico è finito» ${ }^{70}-\mathrm{e}$, considerato il pericolo in cui versava la costruzione comunitaria, si aprivano alle tesi della maggioranza ${ }^{71}$. La quale nel frattempo aveva costituito, proprio in Francia, un Gruppo preparatorio, comprendente anche Spinelli e Serafini, che costituì ufficialmente il Fronte democratico per un'Europa federale, presieduto da Hirsch e particolarmente attivo in occasione delle elezioni legislative francesi del $1967^{72}$. L'unità interna facilitò del resto a sua volta il percorso di avvicinamento, già larvatamente in corso, con le componenti del federalismo e dell'europeismo ad esso esterne, ora più facilmente unificabili dal comune sentimento antigollista e proprio nell'ambito del Fronte. In particolare, si cercava un coordinamento con il Me - il cui Congresso straordinario di Cannes dell'ottobre 1965 aveva espresso viva preoccupazione per la vita

66. Le carte d'archivio documentano non pochi contrasti: cfr. le lettere di J.-P. Gouzy a R. Rifflet (28/03/1963) e a E. Hirsch, A. Marc e R. Rifflet (28/05/1964), in HAEU, RR, 5I/O4 e 53/04. La posizione di Albertini è espressa in Le $X^{e}$ Congres du MFE, IF, vol. VI, n ${ }^{\circ}$ I, 1964, pp. 35 sgg.

67. Cfr. ASUP/FA, LB, 57, MFE-Commission nationale du I4/06/Ig64.

68. GC, vol. I, $\mathrm{n}^{\circ} 2,1965$, p. 2.

69. Cfr. la presa di posizione della Commissione nazionale francese del Mfe (GC, vol. I, no 3, I965, p. 2).

70. GC, vol. II, nº 6, 1966, p. I.

7I. Cfr. M. Albertini, L'opposition en France, IF, vol. VIII, n² 2-3-4, I966, pp. 218 sgg e Id., La renaissance du nationalisme et la lutte du MFE, IF, vol. IX, $\mathrm{n}^{\circ} \mathrm{I}, \mathrm{I} 967, \mathrm{pp} .26 \mathrm{sgg}$.

72. PE, vol. VII, nº 9 , I964, p. 6. 
comunitaria $^{73}$ - e con l'Aef ${ }^{74}$. Fronte e linea unitaria furono sanciti dal Congresso di Torino del 1966, che elesse Albertini alla presidenza del Be e che decise di sostenere apertamente, in vista delle legislative di cui sopra, i partiti dell'opposizione democratica - nella fattispecie il Centro democratico di Lecanuet e la Federazione di Mitterrand - cercando di indirizzarla in senso europeo ${ }^{75}$.

L'ennesimo successo elettorale del Generale, tuttavia, obbligò il Mfe a rivedere ulteriormente la propria strategia: se la sua unità non era mai stata così evidente, mai come in questa fase era del resto emersa anche la mancanza di effettivo peso politico. Si perviene così alla terza e ultima tappa del percorso del Mfe, che si snoda dal 1967 al 1969 e fu interamente volta a ottenere un suo rinnovamento e rafforzamento ${ }^{76}$. Le modalità individuate furono sostanzialmente tre. La prima fu l'adozione in via prioritaria della strategia dell'elezione diretta del Pe, rielaborata da Albertini come proseguimento delle azioni del Cpe e del Censimento — «si tratta di mezzi diversi, in situazioni diverse, per lo stesso fine: l'intervento del popolo nell'integrazione europea» ${ }^{77}$ - e approvata a cavallo delle elezioni francesi in quanto parte, con il Fronte democratico, di uno stesso programma, trasversale a correnti, movimenti e partiti, per «l'urgence de la démocratisation» delle istituzioni europee ${ }^{78}$. Essa, infatti, costituiva non solo il punto di contatto con i partiti democratici - per il motivo molto semplice che «se si chiede che il Pe venga eletto, si chiede una cosa che tutti [...] trovano giusta ${ }^{79}-$ ma, soprattutto, se iniziata in alcuni Paesi con la richiesta di elezioni dirette unilaterali dei rappresentanti nazionali in seno al Pe — come appunto avvenne in Italia — avrebbe acceso una reazione a catena di iniziative collaterali in tutti gli altri, isolando de Gaulle proprio

73. HAEU/AS/Oo64, Circolare di O. Giarini, 25/10/1965.

74. Fu proprio il viaggio a Mosca di de Gaulle a spingere Mfe e Aef a elaborare una presa di posizione comune nel I966, la prima dal 1957 (GC, vol. II, n ${ }^{\circ}$ 7-8, I966, p. 2). Il Cc del Mfe aveva del resto già approvato, nel febbraio 1965, un documento redatto da una Commissione di contatto tra Aef e Mfe creata il 27/0I/1964 (documentazione in ASUP/FA, LB, 57), nel quale si delineava un percorso di avvicinamento basato sulla linea dell'elezione. Il 26 giugno vi fu la prima di una serie di sedute comuni delle due giunte (GC, vol. I, n ${ }^{\circ}$ 2, I965, p. 2); nel 1966 l'Aef fece sua l'idea dell'elezione diretta del Pe e nel 1967, quando tenne il suo primo Congresso, vi invitò i responsabili del Mfe (ASUP/FA, LB, I, Circolari di O. Giarini del 24/or/1967 e del 04/10/1967). Aef e Mfe si riuniranno nel 1973 , ridando vita all'Uef.

75. GC, vol. II, n ${ }^{\circ} 6$, 1966, p. I. I federalisti francesi si erano peraltro schierati pubblicamente per Mitterrand anche in occasione del secondo turno delle elezioni presidenziali del 1965 (HAEU/AS/oo64/o3, Le MFE prend position, $\mathrm{I} 3 / \mathrm{I} 2 / 1965)$.

76. J.-P. Gouzy, La grandezza e il declino, «Federalismo Europeo» (d'ora in poi Fe), vol. I, nº 2, I967, p. I.

77. ASUP/FA, LB, I, Lettera di M. Albertini a L. Bolis, 23/09/1968.

78. ASUPV, LB, I, Compte rendu du CC MFE (II-I2/02/1967) e Compte rendu du CC MFE (oI-02/07/1967).

79. M. Albertini, Un piano d'azione a medio termine, Fe, vol. I, n ${ }^{\circ} 7-8$, I967, p. I. 
sul tema politico della democrazia e del consenso popolare ${ }^{80}$. Sulla base di questa strategia era poi possibile — secondo punto - istituire un coordinamento effettivo a livello di azione con gli altri movimenti e quindi esercitare su di essi una buona dose di influenza politica. Ciò effettivamente avvenne al secondo Congresso dell'Aja del Me (8-9 novembre 1968) ove i federalisti riuscirono a far passare degli emendamenti per la risoluzione finale di natura federale ${ }^{8 \mathrm{r}}$. Ma - terzo e ultimo punto - moltissimo si sperava di ottenere dalle forze coinvolte nel movimento studentesco, che, desiderose di autonomia e partecipazione - i principi base del federalismo - sembravano poter costituire un terreno fertile su cui innestare il seme dell'idea federale, specialmente nella sua versione integrale.

Il '68, come è noto, passò, ma la trasversalità strategica della linea dell'elezione diretta del Pe rimase pur tuttavia valida e portò, al Congresso di Trieste del II-I3 aprile I969, alla sintesi definitiva delle due concezioni del federalismo, integrale e istituzionale: il Mfe si impegnava infatti ad «approfondire e precisare i problemi che condizionano tanto la costruzione europea che l'edificazione di una società federale» ${ }^{82}$. Poco dopo la caduta di de Gaulle, fu poi deciso dal Cc di «compiere tutti i passi necessari per unificare le organizzazioni federaliste, dimenticando le dispute del passato e tenendo in vista solo le lotte dell'avvenire» ${ }^{83}$, inserendo la lotta per l'elezione in un disegno politico globale, che proponeva a tutti i partigiani dell'Europa un'azione comune di agitazione e pressione.

In tale seduta del Cc Spinelli, appena ridiventatone membro, aveva elencato gli obiettivi sui quali i federalisti avrebbero dovuto concentrarsi da allora in avanti: adesione inglese, fine del periodo transitorio, allargamento alla comunità politica affidata al Pe, elezioni europee, alleanza con le forze europeiste. Il commento che egli affida ai diari costituisce una delle più eloquenti testimonianze della portata del cambiamento avvenuto nell'arco di poco più di un decennio in seno al Mfe-sovrannazionale, che riuscì a superare divisioni e crisi di orientamento politico — in ciò sospinto, come si è visto, dalle politiche di de Gaulle - acquisendo la forza e la coerenza che caratterizzeranno la sua azione politica nel quindicennio successivo: «Con mio stupore tutti hanno accettato tutto» ${ }^{84}$.

8o. ASUP/FA, LB, I, Lettera di M. Albertini ai membri del CC, s. d.

8I. Cfr. ASUP/FA, LB, I, Circolare di O. Giarini, I4/II/I968.

82. Vedi Fe, vol. III, n 2 , 1969.

83. Ivi, p. 3.

84. A. Spinelli, Diario europeo, cit., p. 552. 